\section{Pathophysiology of Haemostasis and Thrombosis}

Pathophysiol Haemost Thromb 2005;34:135-142

DOI: $\underline{10.1159 / 000089933}$

\title{
Blood Coagulation and Propagation of Autowaves in Flow
}

\author{
Elena A. Ermakova ${ }^{a}$ Mikhail A. Panteleev ${ }^{c}$ Emmanuil E. Shnol ${ }^{\text {b }}$ \\ a Semenov Institute of Chemical Physics, Russian Academy of Sciences, Moscow, \\ ${ }^{b}$ Institute of Mathematical Problems of Biology, Russian Academy of Sciences, Pushchino, Moscow Region, and \\ ${ }^{c}$ National Research Center for Hematology, Russian Academy of Medical Sciences, Moscow, Russia
}

\section{Key Words}

Blood coagulation - Autowaves · Blood flow •

Thrombomodulin

\begin{abstract}
This study analyses the effect of flow and boundary reactions on spatial propagation of waves of blood coagulation. A simple model of coagulation in plasma consisting of three differential reaction-diffusion equations was used for numerical simulations. The vessel was simulated as a two-dimensional channel of constant width, and the anticoagulant influence of thrombomodulin present on the undamaged vessel wall was taken into account. The results of the simulations showed that this inhibition could stop the coagulation process in the absence of flow in narrow channels. For the used mathematical model of coagulation this was the case if the width was below $0.2 \mathrm{~mm}$. In wider vessels, the process could be stopped by the rapid blood flow. The required flow rate increased with the increase of the damage region size. For example, in a $0.5-\mathrm{mm}$ wide channel with 1-mm long damage region, the propagation of coagulation may be terminated at the flow rate of more than $20 \mathrm{~mm} / \mathrm{min}$.
\end{abstract}

\section{Introduction}

\section{Blood Coagulation}

The complex process of coagulation involves numerous proteins and specialized blood cells, platelets. Plasma coagulation is a cascade of enzymatic reactions (for a recent review, see [1]), ultimately catalyzing fibrinogen conversion into fibrin, which polymerizes to form a clot. This can occur without platelet plug formation, and the present study is focused on the formation of these fibrin clots.

The coagulation cascade involves multiple feedback loops, and of particular interest is the positive feedback loop of factor XI activation by thrombin: this reaction allows autocatalytic thrombin generation far from the damage site and can lead to an autowave-like expansion of the clotting process [2]. The spatial fibrin clot formation in the vessel could be differentiated into three stages [3]: (1) initiation (localized at the vascular damage site); (2) propagation, or spatial expansion of coagulation wave into the vessel; and (3) termination of the biochemical reactions and clot enlargement stoppage.

The propagation stage is similar to other nonlinear wave processes. When studying this stage, it is possible to substitute the complex initiation process with appropriate initial conditions stimulating the autowave formation. For example, it is possible to set a sufficiently high (i.e., above-threshold) concentration of thrombin, the

\begin{tabular}{ll}
\hline KARGER & ( ) 2005 S. Karger AG, Basel \\
Fax +4161306 1234-8832/05/0343-0135\$22.00/0 \\
$\begin{array}{l}\text { E-Mail karger@karger.ch } \\
\text { www.karger.com }\end{array}$ & $\begin{array}{l}\text { Accessible online at: } \\
\text { www.karger.com/pht }\end{array}$
\end{tabular}


principal component of coagulation system, in a thin region adjacent to the damage site. The mechanisms of the termination stage are not completely understood. In particular, it has been suggested that blood flow removing active coagulation factors provides the localization of fibrin clot in the region of vessel wall damage [4].

The process of blood coagulation has many distinctive properties of the autowave processes [5], the most important of them being the fact that the velocity of the clotting front propagation at the second stage is independent of the initiation method [6]. On the other hand, the process of clotting propagation has several properties distinguishing it from the majority of autowave phenomena. First, due to obvious physiological reasons, this process should be always stopped in vivo despite the presence of free (able to clot) plasma medium. Second, this process occurs under conditions of flow. Finally, the boundaries of the region, where coagulation occurs, are active: the damaged vessel wall activates coagulation, while intact endothelium inhibits it via expression of thrombomodulin and other substances.

Interaction of autowaves with flows and effects of 'active' boundaries are phenomena of general interest, not limited to the blood coagulation. We studied them in a simple model of blood coagulation (three differential equations) obtained earlier [7] as a result of modification and reduction of a more detailed model of the coagulation cascade [8].

\section{Autowaves}

There are numerous processes in physics, chemistry, and biology, which are called 'autowave processes' [5, 911]. The most well-known ones are flame and neural pulse propagation. The former problem can be modeled mathematically using a single reaction-diffusion differential equation:

$$
\frac{\partial u}{\partial t}=f(u)+d \Delta u ; \quad \Delta=\frac{\partial^{2}}{\partial x^{2}}+\frac{\partial^{2}}{\partial y^{2}}+\frac{\partial^{2}}{\partial z^{2}},
$$

where $t$ is time, $x, y$, and $z$ are coordinates, and $\Delta$ is Laplacian. The $u$ variable can be either temperature or concentration of a component participating in the complex of chemical reactions [12]. The first item in the right part of Eq 1 describes processes occurring in each element of the medium, while the second one describes a linear diffusion-like interaction between the elements. The theory of neural pulse propagation is based on the model of Hodgkin and Huxley comprising four equations [9].

For both cases, one-dimensional problems are usually studied (then $\Delta$ is transformed into $\partial^{2} / \partial x^{2}$ ). In addition, research is usually focused on steady, established regimes, which do not depend on the 'firing' or 'excitation' stage. These regimes are qualitatively different for these two cases: flame propagation is a trigger wave of irreversible transition; neural pulse is a localized wave of excitation, after passing of which the medium returns to the initial excitable state.

The steady one-dimensional waves and respective plane waves in a two- or three-dimensional medium give the most obvious demonstration of the autowave nature of these processes: their amplitude, shape, and velocity do not depend on the initial conditions. Mathematical modeling of an excitable medium requires at least two equations of the Eq 1 type. One of the variables describes the excitation itself, and another describes the inhibition of this excitation and recovery of the medium; these processes have usually different time scales. A simple and the most well-known model of an excitable medium, that of Fitz Hugh-Nagumo is often used [5]. The model of blood coagulation (Eq 3 ) used in this work also describes an excitable medium.

There are a limited number of studies of autowaves in moving media. We shall mention two of them. The work [13] analyzes a model of flow reactor in the form of a twodimensional channel with one irreversible reaction. The flow in the channel is parabolic so that the velocity is maximal in the middle and is zero at the walls; the reaction is activated at the beginning of the channel. Under influence of flow, the plane front of the reaction wave bends and ultimately moves at a constant velocity keeping this shape. Mathematical description of the problem gives a single partial differential equation of the Eq 1 type, which is solved to obtain trigger wave solutions. In numerical experiments using this equation, the authors observed the above course of events in a wide range of rates in parabolic and linear flows.

Plane excitation waves in a two-dimensional active medium were studied in [14]. The medium was assumed to be infinite and to move along the $x$ axis, and the velocity was assumed to have a constant gradient $a$, i.e., to follow the function $V(y)=a y$. The flow changed the direction and the slope of wave front (i.e., the level curves of all variables), though the wave remained plane. The authors show that there is a threshold value $a^{*}$ of flow nonuniformity. At $a<a^{*}$, a plane wave can exist, being tilted to the direction of flow, while at $a>a^{*}$ the wave becomes too 'narrow', and the excitation disappears. Using the Fitz Hugh-Nagumo model, they estimate the value of $a^{*}$ analytically and show its correlation with the results of numerical experiments. The simulations were necessarily 
performed for a finite region; the effects of boundaries were not discussed.

The difference between these two studies should be noted. Stationary trigger waves [13] seem to exist in the model analyzed for all flow rates. On the contrary, stationary excitation pulses do not exist at high $V^{\prime}(y)$ values.

\section{Blood Coagulation Model}

Propagation of autowaves in the models of blood coagulation in the absence of flow has been a subject of several studies [7, 15, 16]. However, the in vivo clot formation is a process occurring in the presence of flow, and the role of flow appears to be essential. An attempt to study the effects of slow flow on thrombus formation were made $[17,18]$, where a phenomenological model of coagulation was used. In the present study, we utilized a simple mathematical model containing three differential equations. This model is a reduced form of a larger model based on the biochemistry of blood coagulation cascade [7]. The model is designed to describe the second, propagation stage of blood coagulation. There is no fibrin in the model, although it is possible to assume in the interpretation of the simulations below that polymerization directly follows the thrombin wave. The model is designed to describe processes with characteristic times less than an hour: for example, it does not contain fibrinolysis reactions and thus prolonged simulations could not be physiologically interpreted.

\section{Equations and Initial Conditions}

We consider a rectangular zone on a $(x, y)$ plane with a width of $H(0 \leq y \leq H)$, with the medium moving along the $x$ axis with a velocity of $V(y)$. We assume a parabolic velocity profile corresponding to the laminar flow of viscous incompressible fluid:

$$
V(y)=a y(1-y / H), a>0, \quad V_{\max }=1 / 4 a H .
$$

To make the comparison simpler, we shall keep the notation of 'biochemical' terms used in [7], adding only a multiplier to take into account the difference in the units of time:

$$
\begin{aligned}
& \frac{\partial u_{1}}{\partial t}=\frac{1}{\tau_{1}}\left[K_{1} u_{1} u_{2}\left(1-u_{1}\right) \frac{1+K_{2} u_{1}}{1+K_{3} u_{3}}-u_{1}\right]+D \cdot \Delta u_{1}+V(y) \frac{\partial u_{1}}{\partial x} \\
& \frac{\partial u_{2}}{\partial t}=\frac{1}{\tau_{1}}\left[u_{1}-K_{4} u_{2}\right]+D \cdot \Delta u_{2}+V(y) \frac{\partial u_{2}}{\partial x} ; \quad \Delta=\frac{\partial^{2}}{\partial x^{2}}+\frac{\partial^{2}}{\partial y^{2}} \\
& \frac{\partial u_{3}}{\partial t}=\frac{1}{\tau_{1}}\left[K_{5} u_{1}^{2}-K_{6} u_{3}\right]+D \cdot \Delta u_{3}+V(y) \frac{\partial u_{3}}{\partial x}
\end{aligned}
$$

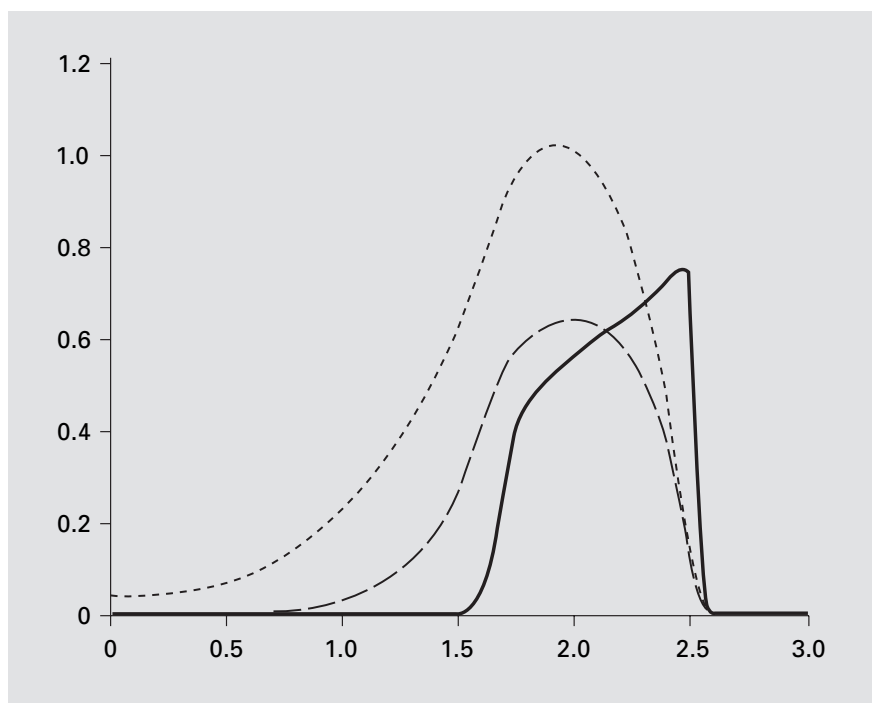

Fig. 1. The shapes of the functions in the reduced model of blood coagulation used in this study. Shown are concentration profiles of: $u_{1}(x)$, thrombin (bold line); $u_{2}(x) / 10$, activated factor XI (dashed line); $u_{3}(x) / 100$, activated protein $\mathrm{C}$ (dotted line).

Table 1. Parameters of the blood coagulation model ${ }^{\mathrm{a}}$

\begin{tabular}{lllllll}
\hline$K_{1}$ & $K_{2}$ & $K_{3}$ & $K_{4}$ & $K_{5}$ & $K_{6}$ & $D, \mathrm{~mm}^{2} / \mathrm{min}$ \\
\hline 6.85 & 20.0 & 2.36 & 0.087 & 15.0 & 0.04 & 0.0006 \\
\hline
\end{tabular}

${ }^{\text {a }}$ The parameters are from [7] with minor changes.

where dimensionless variables $u_{1}, u_{2}$, and $u_{3}$ represent concentrations of thrombin (IIa), activated factor XI (XIa), and activated protein $\mathrm{C}(\mathrm{PCa})$. They are linked with the dimensional concentrations as follows:

$$
\begin{aligned}
& {[I I a]=10^{3} u_{1} \mathrm{n} M, \quad[X I a]=0.043 u_{2} \mathrm{n} M,} \\
& {[P C a]=0.61 u_{3} \mathrm{n} M .}
\end{aligned}
$$

The dimensionless coefficients $K_{i}$ are combinations of kinetic constants of the reactions and typical factor concentrations in the initial (not reduced) set of equations [7]. $\tau_{1}$ is characteristic time of thrombin inactivation. If time $t$ has dimensionality of minutes, and distance is in millimeters, then $\tau_{1}=0.43 \mathrm{~min}$, and diffusion coefficients $D$ for these three factors are assumed to be equal to 0.0006 $\mathrm{mm}^{2} / \mathrm{min}$. Note that a dimensionless variable $t / \tau_{1}$ was used for the time in article [7].

The values of model parameters used for the simulations below are summarized in table 1; they are slightly 
different from those used in [7]. For these values, in the one-dimensional model there are stable excitation pulses with constant shape and velocity $v: u_{i}(x, t)=$ $\phi_{i}(x-v t), v>0$. These pulses are shown in figure 1 . If the medium is motionless and the boundary conditions are nonpermeable ( $u_{y}^{\prime}=0$ for $\left.y=0, H\right)$, then the same formulae result in a solution of Eq 3 in the form of plane waves moving along the channel. For values used in figure 1, the velocity is $v=0.045 \mathrm{~mm} / \mathrm{min}$ (in agreement with the experimental data [6]), $u_{1 \max }=0.76$. As width $L_{p}$ of this wave we denote the distance between the level lines $u_{1}=$ $0.08\left(\approx 0.1 u_{1 \mathrm{max}}\right)$. For the selected parameters, $L_{p}=$ $0.8 \mathrm{~mm}$. It should be noted that physiological vessel diameters can be often smaller than $L_{p}$. For $t=0$, the initial conditions are set in the following way:

$$
\begin{aligned}
& u_{1}(x, y)=u_{1}^{0} \text { within a rectangle } \\
& P=\left\{x_{1} \leq x \leq x_{2}, 0 \leq y \leq l_{y}\right\}, x_{2}-x_{1}=l_{x} \\
& u_{1}(x, y)=0 \text { outside of } P, \quad u_{2}=u_{3}=0 \text { everywhere. }
\end{aligned}
$$

The interval from $x_{1}$ to $x_{2}$ imitates the damaged region of the vessel wall, and setting a fixed thrombin concentration in $P$ mimics the activation process of blood coagulation. The rectangle $P$ will be called the initial excitation region.

\section{Vessel Wall Reactions and Active Boundary Conditions}

The 'active' boundary conditions describe the thrombomodulin-dependent anticoagulant reactions of the protein $\mathrm{C}$ pathway occurring on the intact vessel wall (see Appendix). For the 'damaged' region $\left(y=0 ; x_{1}<x<x_{2}\right)$ standard nonpermeability conditions are used. To make the conditions biochemically adequate, we use additional variable $s$, defined only on the boundary, which represents surface density of thrombomodulin. For a given $x$ and for $y=0$ or $y=H$, the evolution of this variable is determined by the ordinary differential equation:

$$
\frac{\partial s}{\partial t}=-k_{a} s \cdot[I I a] \cdot+k_{d} \cdot\left(s^{0}-s\right)
$$

where $k_{a}, k_{d}, s^{0}$ are constants (see Appendix). Eq 6 for the bottom boundary $(y=0)$ is valid outside of the 'damaged' region $\left(x<x_{1}\right.$ or $\left.x>x_{2}\right)$; for the top boundary, it is valid for all $x$ values. Boundary conditions result in the decrease of thrombin concentration and increase of activated protein $\mathrm{C}$ concentration near the intact vessel walls. Using a dimensionless variable $\sigma=s / s^{0}$ and Eq 4, equation 6 is transformed into:

$$
\frac{\partial \sigma}{\partial t}=-\widetilde{k}_{a} \sigma u_{1}+k_{d}(1-\sigma) .
$$

Table 2. Parameters of the boundary conditions in blood coagulation model

\begin{tabular}{lllll}
\hline$\tilde{k}_{a}$ & $k_{d}$ & $\gamma_{1}$ & $\gamma_{2}$ & $\gamma_{3}$ \\
\hline $500 \mathrm{~min}^{-1}$ & $0.25 \mathrm{~min}^{-1}$ & $167 \mathrm{~mm}^{-1}$ & $0.083 \mathrm{~mm}^{-1}$ & $1,017 \mathrm{~mm}^{-1}$ \\
\hline
\end{tabular}

Boundary conditions for the set (Eq 3$)$ at $y=0$ outside of the 'damage site' are:

$$
\frac{\partial u_{1}}{\partial y}=\gamma_{1} \cdot \sigma u_{1}-\gamma_{2}(1-\sigma) ; \quad \frac{\partial u_{3}}{\partial y}=-\gamma_{3}(1-\sigma) ; \quad \frac{\partial u_{2}}{\partial y}=0 .
$$

Within the 'damage site':

$$
\frac{\partial u_{k}}{\partial y}=0 \text { for } x_{1} \leq x \leq x_{2}, \quad y=0 ; \quad k=1,2,3 .
$$

Boundary conditions for $y=H$ are obtained from Eq 8a upon the change of the sign in the right parts. The coefficient values used in equations 6-8 are listed in table 2 (see also Appendix).

\section{Results}

\section{Action of Thrombomodulin in the Absence of Flow}

Although it is obvious that the anticoagulant processes on the channel wall hamper the coagulation propagation, the manner of inhibition and its significance for different physiological conditions are unclear. Our simulations suggest that thrombomodulin located on the channel wall at physiological concentrations is able to stop thrombin propagation in a narrow channel. For a small $H$ and boundary conditions (equations $6-8$ ) the initial activation (Eq 5) disappears after some period of time and all variables return to the equilibrium values $u_{i}=0$. Figure 2 illustrates the process of excitation for $H=0.23 \mathrm{~mm}$ in the absence of flow ( $a=0$ in Eq 2).

The excitation does not expand to the sides, because of the inhibition by intact wall and is suppressed in 20 min near the activation region. There is a maximal channel width $H^{*}$, where this scenario exists. For $H>H_{*}$, the excitation does not disappear, and two identical waves moving to the left and to the right are formed. The inhibiting boundary conditions inhibit excitation near the walls, and these waves are not flat: functions $u_{i}$ essentially depend on $y$. 


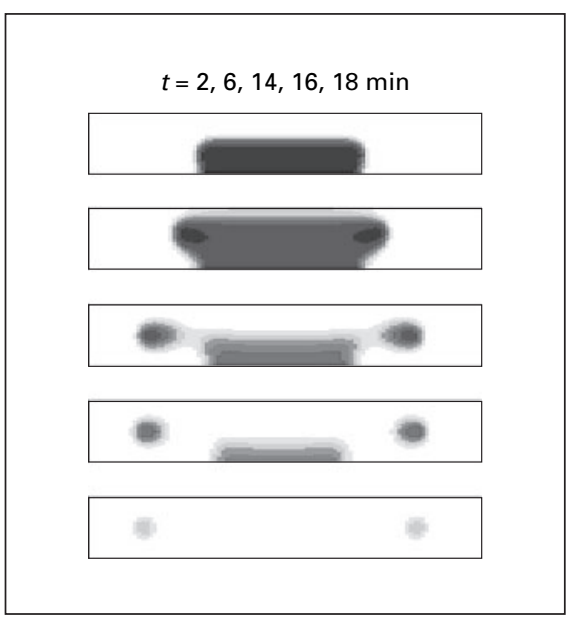

Fig. 2. Disappearing of the excitation in a narrow channel in the absence of flow due to the action of thrombomodulin. The activation rectangle region is seen in the bottom part of the simulation region $l_{x} \times l_{y}=0.8 \mathrm{~mm} \times 0.05 \mathrm{~mm} ; u_{1}^{(0)}=0.5$. The simulation region size is $L=2 \mathrm{~mm}, H=0.23 \mathrm{~mm}$. The series of images show the region at $t=2,6,14,16$, and $18 \mathrm{~min}$ (top to bottom). At $t=20 \mathrm{~min}$, the excitation disappeared.

\section{Effects of Small Flow Rates}

Let the channel width $H$ be fixed at $0.5 \mathrm{~mm}$, which approximately 2 -fold exceeds the critical value $H^{*}$. At small flow rates, the system evolution is similar to that described above (not shown), though the shapes of these two waves are no more identical. When the flow rate becomes comparable to the wave velocity $v$, almost stationary structures are observed (fig. 3). The forward front of this structure is directed opposite to the flow; the second wave is carried away by flow. The range of flow rates where this is observed depends on the size of the stimulation region. For $l_{x}=0.5 \mathrm{~mm}, l_{y}=0.05 \mathrm{~mm}, u^{0}=0.5$, this phenomenon is observed in the range of $0.056<V_{\max }<0.17 \mathrm{~mm} / \mathrm{min}$ $\left(1.2<V_{\max } / v<3.7\right)$. Increase of the activation region allows these structures to exist at higher flow rates.

One of the possible scenarios observed at $V_{\max }$ exceeding the wave velocity $v$ is shown in figure 4 . It shows formation of a single stable wave moving along the flow axis at a velocity of $1.44 \mathrm{~mm} / \mathrm{min} \approx v+V_{\max }$. The final shape of the wave depends on the flow rate. In particular, its maximal width along the $x$ axis increases with the increase of flow rate. For example, the wave width at $V_{\max }=0.19$ and $1.4 \mathrm{~mm} / \mathrm{min}\left(V_{\max } / v=3.7\right.$ and 30 , respectively) is 1.3 and $6 \mathrm{~mm}$, respectively.

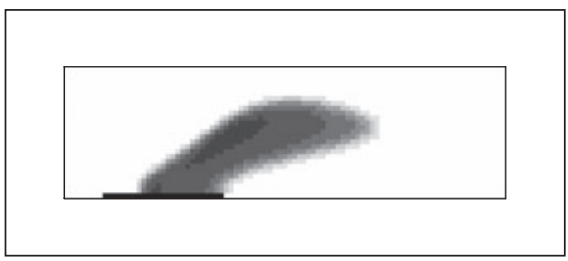

Fig. 3. Stationary structure in moving medium. The current is left to right. The channel width is $H=0.5 \mathrm{~mm}, V_{\max }=0.056 \mathrm{~mm} / \mathrm{min}$. The forward front of the structure is to the left. The dark line in the bottom shows the damaged wall length: $l_{x}=0.5 \mathrm{~mm}$.

\section{Excitation Disappearance at High Flow Rates}

The minimal flow rate $V^{*}$ leading to the stopping of excitation is a function of initial conditions. In particular, if activation region width $l_{\mathrm{y}}$ and activation intensity $u_{1}^{0}$ are fixed, then $V^{*}$ increases with the increase of activation region length $l_{x}$. In other words, in order for initial excitation to sustain, the activation zone should be increased or the flow rate should be decreased. This is in agreement with the results obtained earlier [19] for an autocatalytic system on cell membrane under flow conditions. They studied small spatial and temporal scales (approximately by one or two orders of magnitude smaller than in this work) and found that increase of the membrane patch size and decrease of the flow velocity help to lower the activation threshold. In contrast to our simulations, the model [19] is spatially one-dimensional; it does not contain diffusion to the surface and there is a non-zero flow velocity at the membrane (while in a viscous medium the wall velocity is zero).

There are two principal modes of evolution of the initial excitation. The coagulation wave can either quickly disappear, remaining in the high flow gradient region or reach the middle of the stream and remain there for a long time. Figure 5 illustrates both these possibilities. It is simulated for $H=0.5 \mathrm{~mm}$ and $l_{x}=1 \mathrm{~mm}, l_{y}=0.05 \mathrm{~mm}$, $u^{0}=0.5$. At $V_{\max }=18 \mathrm{~mm} / \mathrm{min}$ (fig. $5 \mathrm{a}$ ) the excitation rapidly disappears after a small period of time: $u_{1}$ was below 0.01 after $t>1.5$. At $V_{\max }=12 \mathrm{~mm} / \mathrm{min}$ the excitation existed for a long time. The excited region leaves the activation zone and moves with flow slowly expanding and increasing $u_{1}$ (fig. 5b). If $l_{x}$ is decreased 2-fold at $V_{\max }=12 \mathrm{~mm} / \mathrm{min}$, the excitation disappears as rapidly as in figure 5a. Numerous simulations at other parameter values (not shown) also confirm the above statement: the larger the excitation region, the higher the flow rate required to suppress the excitation and localize it to the activation zone.

Pathophysiol Haemost Thromb 2005;34:135-142 
Fig. 4. Stationary waves in moving medium (blood coagulation model). The current is left to right. a $V_{\max }=0.19 \mathrm{~mm} / \mathrm{min}$, the wavelength along the axis is $1.3 \mathrm{~mm}$; b $V_{\max }=1.4 \mathrm{~mm} / \mathrm{min}$, the wavelength along the axis is $6 \mathrm{~mm}$. For better presentation, the $y$ scale is 5-fold smaller than the $x$ scale.
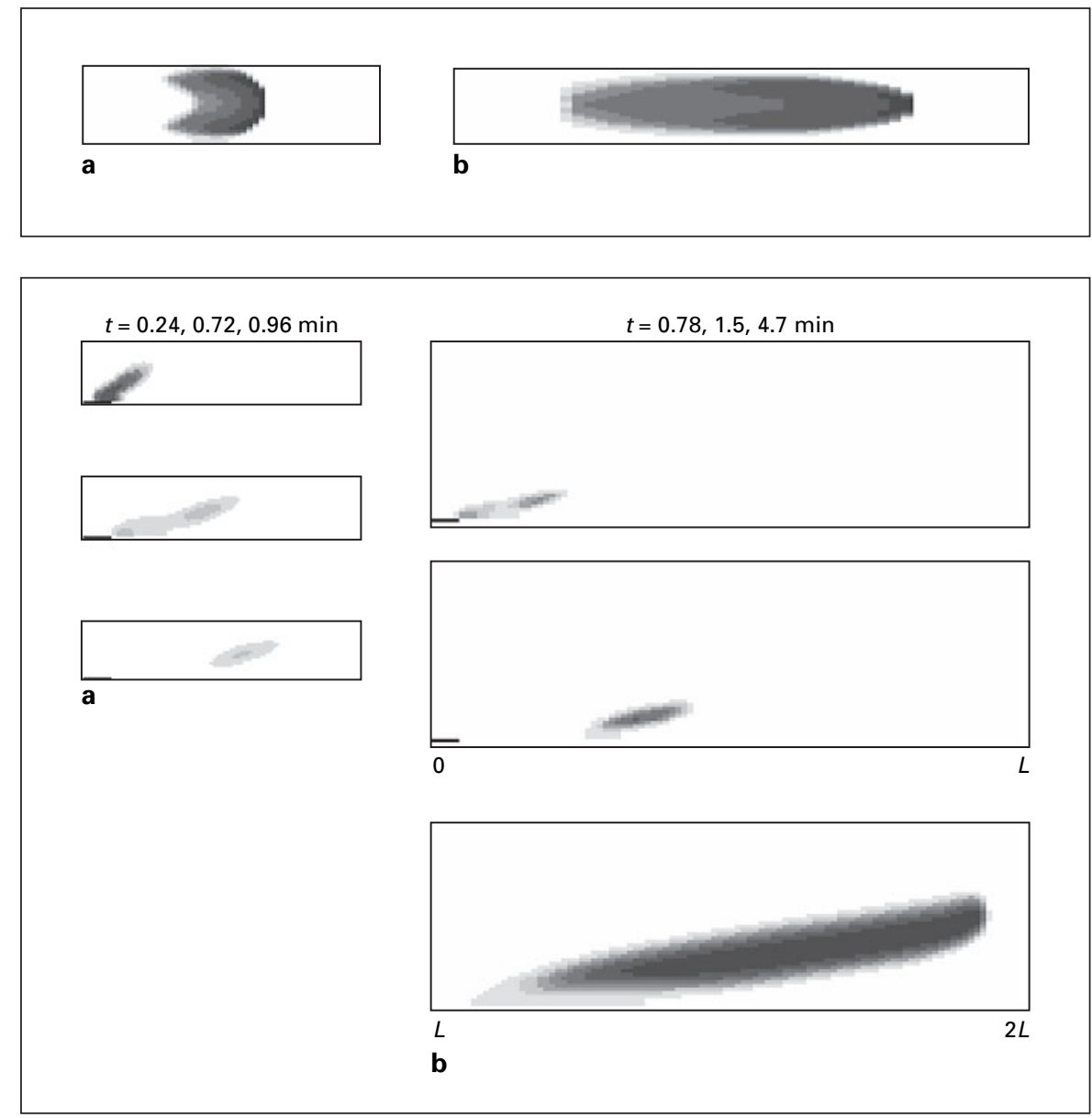

Fig. 5. The effect of flow rate on the survival of thrombin autowave. The current is left to right. The dark line in the bottom shows the damaged wall length. The activation region: $l_{x} \times l_{y}=1 \mathrm{~mm} \times 0.05 \mathrm{~mm}$. a $V_{\max }=18.7 \mathrm{~mm} / \mathrm{min} ; \mathbf{b} V_{\max }=12.5 \mathrm{~mm} /$ min. $L=10$ for $\mathbf{a}, L=20$ for $\mathbf{b}$. The series of images show the simulated region at $t=0.24,0.72$, and $0.96 \mathrm{~min}$ in a (a 20\% bottom section of the region is shown) and at $t=0.78,1.5$, and $4.7 \mathrm{~min}$ in $\mathbf{b}$ (top to bottom).

\section{Concluding Remarks}

We analyzed a simple blood coagulation model (three differential equations). We were interested in the effects of nonuniform flow and active boundaries on the coagulation wave propagation in two-dimensional channels.

We used reaction schemes and kinetic parameters reported in the experimental literature and were able to obtain quantitative data: the inhibiting influence of thrombomodulin on intact vessel wall suppresses the autowave development at channel width close to the diameters of relatively large vessels, such as arterioles $(\approx 0.25 \mathrm{~mm})$.

In contrast to trigger waves, which correspond to irreversible chemical reactions, excitation autowaves cannot exist at high gradients of flow and disappear. This has been shown [14] for plane waves and constant flow rate gradient. We observed suppression of excitation by rapid flow. Noteworthy, for a parabolic flow there is a low-gradient zone near the axis of the flow. Due to this, excitation waves can exist in narrow channels even at high flow rate gradients near the walls. Additional stabilizing effect is produced by 'passive' boundary conditions (no interactions of proteins with the wall).

For a given channel, there is no general value of the threshold flow rate or near-wall gradient: the events depend on the initial conditions and boundary conditions on vessel wall. The evolution of the initial excitation at low flow rates can be rather different. In particular, excitation waves with constant velocity and shape can appear despite the nonuniformity of flow. This remarkable synchronization of wave processes at different flow lines was first reported for a chemical autowave described by a single equation $[13,20-22]$. The moving force of this synchronization is the diffusion interaction between parallel lines of flow having different velocities. As noted above, excitation processes can exist only if the flow nonuniformity is not very high. We do not know whether a nonsuppressed initial excitation always results in stationary waves. 
The model of blood coagulation used does not describe fibrin clot formation. An accurate quantitative description of this process requires more complex mathematical models and is beyond the scope of this work. Additional simulations with more complex and detailed biochemical model are also required to estimate possible significance and interpretation of thrombin waves following the flow far from the damage site. It should be also noted that biochemical reactions and diffusion, if not convection, still occur within a fibrin clot. However, addition of the fibrin clot formation (which changes the flow pattern) and the cylindrical vessels into the model seem unlikely to affect two general conclusions of this study: that clotting process can be stopped by the inhibiting influence of intact vessel walls and rapid flow.

\section{Acknowledgements}

We thank V.N. Biktashev, A.B. Medvinskii, O.A. Mornev, and M.A. Tsyganov for helpful discussion of autowave problems and for pointing out literature references. We are grateful to A.M. Zhabotinsky, Yu.M. Romanovskii, and H. Malchow for valuable references. We express our special gratitude to F.I. Ataullakhanov for his counsel and important discussions during this work. The study was supported in part by grants No. 03-04-48338 and 05-0122001 from Russian Foundation for Basic Research.

\section{Appendix: Active Boundary Conditions to Describe Interaction of Proteins with Vessel Wall}

\section{Biochemistry of Blood Coagulation Factors' Interaction with Endothelium}

To perform computer simulations of coagulation in vessel with thrombomodulin molecules present on intact endothelium, we use modified boundary conditions as described in the following sections. The model takes into account the presence of thrombomodulin, the thrombin-thrombomodulin complex formation, and protein $\mathrm{C}$ activation by this complex. This section describes and comments the inclusion of these processes into the mathematical model of this study.

The following assumptions were used. We assumed the vessel wall, except for the damaged region, to be uniformly covered with thrombomodulin at a surface density of $s^{0}=2 \times 10^{-7} \mathrm{nmol} / \mathrm{mm}^{2}$ (assuming $\sim 40,000$ thrombomodulin molecules per endotheliocyte [23], half of which are in contact with plasma, and estimating the density of cells in endothelium as $\sim 6,000$ cells $/ \mathrm{mm}^{2}$ ). We assume thrombin to bind thrombomodulin with equilibrium constant $K_{d}=0.5 \mathrm{n} M$ [24], the rate constants being $k_{a}=0.5 \mathrm{n} M^{-1} \mathrm{~min}^{-1}$; $k_{d}=0.25 \mathrm{~min}^{-1}$. The resulting complex activates protein $\mathrm{C}$ following the Michaelis kinetics with $k_{\text {cat }}=250 \mathrm{~min}^{-1}, K_{M}=8,000 \mathrm{n} M$ [25].

\section{Notation and Dimensionality}

Let $y$ be the distance perpendicular to the channel wall. Thrombomodulin is located at $y=0$. We also denote: $s$ is the surface density of thrombomodulin; $s^{0}-s$ is the thrombin-thrombomodulin complex surface density; $s^{0}=$ const is the initial density of thrombomodulin; $[I I a]$ is the volume concentration of thrombin; $[\mathrm{PCa}]$ is the concentration of activated protein $\mathrm{C}$. We shall express surface density in nanomoles per square millimeters, the distance along the $y$ axis will be in millimeters, the volume concentration will be in nanomoles per litre $(\mathrm{n} M)$, the time will be in minutes, and diffusion coefficients will be in square millimeters per minute.

\section{Changes in the Model}

The reaction-diffusion equations of the model do not change. However, an additional function $s$, defined only on the vessel wall, appears. In addition, the boundary conditions for coagulation proteins interacting with endothelium are changed in comparison with the nonpermeability case.

The variable $s$ changes because of thrombin binding to thrombomodulin from plasma:

$$
\frac{\partial s}{\partial t}=-\left.k_{a} \cdot s \cdot[I I a]\right|_{y=0}+k_{d} \cdot\left(s^{0}-s\right) .
$$

Thrombin interacts with thrombomodulin resulting in the changes of the boundary conditions at $y=0$ :

$$
\left.D_{I I a} \cdot \frac{\partial[I I a]}{\partial y}\right|_{y=0}=-10^{6} \cdot\left(-k_{a} \cdot s \cdot[I I a]_{y=0}+k_{d} \cdot\left(s^{0}-s\right)\right) \text {. }
$$

Activated protein $\mathrm{C}$ is produced by the thrombin-thrombomodulin complex:

$$
\begin{aligned}
& \left.D_{P C a} \cdot \frac{\partial[P C a]}{\partial y}\right|_{y=0}=-10^{6} \cdot \frac{k_{c a t} \cdot[P C] \cdot\left(s^{0}-s\right)}{K_{M}+[P C]}= \\
& -10^{6} \cdot k_{e f f} \cdot\left(s^{0}-s\right) .
\end{aligned}
$$

The mean plasma concentration of protein $\mathrm{C}$ is $[\mathrm{PC}]=60 \mathrm{n} M$, and therefore $k_{\text {eff }}=1.86 \mathrm{~min}^{-1}$. The coefficients $10^{6}$ in equations $\mathrm{A} 2$ and $\mathrm{A} 3$ are a consequence of the difference in used units of length and volume. On the left, there are diffusion currents, which are in $\mathrm{mm}^{2} / \mathrm{min} \times \mathrm{nmol} / \mathrm{liter} / \mathrm{mm}=10^{-6} \mathrm{nmol} / \mathrm{mm}^{2} / \mathrm{min}$. On the right, there are reaction currents which are in $1 / \min \times(\mathrm{n} M) \times$ $\left(\mathrm{nmol} / \mathrm{mm}^{2}\right) /(\mathrm{n} M)=\mathrm{nmol} / \mathrm{mm}^{2} / \mathrm{min}$. Thus, the unit of measure of the quantities in the left part is $10^{-6}$ of the quantities in the right part. Therefore, to find numerical values of the quantities in the left part it is necessary to multiply right parts by $10^{6}=1$ iter $/ \mathrm{mm}^{3}$. 


\section{References}

$\checkmark 1$ Schenone M, Furie BC, Furie B: The blood coagulation cascade. Curr Opin Hematol 2004; 11:272-277.

$\checkmark 2$ Zarnitsina VI, Pokhilko AV, Ataullakhanov FI: A mathematical model for the spatio-temporal dynamics of intrinsic pathway of blood coagulation. II. Results. Thromb Res 1996;84: 333-344.

3 Ataullakhanov FI, Zarnitsyna VI, Kondratovich AYu, Lobanova ES, Sarbash VI: A new class of stopping self-sustained waves: a factor determining the spatial dynamics of blood coagulation. Physics - Uspekhi 2002;45:619_ 636.

4 Barynin YaA, Starkov IA, Khanin MA: Mathematical models in hemostasis physiology. Biology Bulletin 1999;47-54.

5 Vasiliev VA, Romanovskii YuM, Chernavskii DS, Yakhno VG: Autowave Processes in Kinetic Systems: Spatial and Temporal Selforganization in Physics, Chemistry, Biology and Medicine. Dordrecht/Boston/Lancaster/ Tokyo, Reidel Publishing Company, 1987.

-6 Ovanesov MV, Krasotkina JV, Ul'yanova LI, Abushinova KV, Plyushch OP, Domogatskii SP, Vorob'ev AI, Ataullakhanov FI: Hemophilia A and B are associated with abnormal spatial dynamics of clot growth. Biochim Biophys Acta 2002;1572:45-57.

-7 Zarnitsina VI, Ataullakhanov FI, Lobanov AI, Morozova OL: Dynamics of spatially nonuniformig patterning in the model of blood coagulation. Chaos 2001;11:57-70.
$D_{8}$ Zarnitsina VI, Pokhilko AV, Ataullakhanov FI: A mathematical model for the spatio-temporal dynamics of intrinsic pathway of blood coagulation. I. The model description. Thromb Res 1996;84:225-236.

9 Zykov VS: Simulation of Wave Processes in Excitable Media. Manchester, Manchester University Press, 1988.

10 Mikhailov AS: Foundations of Synergetics. I. Distributed Active Systems, ed 2, revised. Berlin, Springer, 1994.

11 Zhabotinsky AM: Concentration Autooscillations (in Russian). Moscow, Nauka, 1974.

12 Frank-Kamenetskii DA: Diffusion and Heat Transfer in Chemical Kinetics. New York, Plenum Press, 1969.

13 Allen MA, Brindley J, Merkin JH, Pilling MJ: Autocatalysis in a shear flow. Phys Rev E 1996; 54:2140-2142.

14 Biktashev VN, Biktasheva IV, Holden AV, Tsyganov MA, Brindley J, Hill NA: Effect of shear flows on nonlinear waves in excitable media. J Biol Phys 1999;25:101-113.

15 Ataullakhanov FI, Guriya GT: Spatial aspects of the dynamics of blood clotting. I. Hypothesis. Biophysics (Engl. Transl.) 1994;39:91-97.

16 Ataullakhanov FI, Guriya GT, Safroshkina AYu: Spatial aspects of the dynamics of blood clotting. II. Phenomenological model. Biophysics (Engl. Transl.) 1994;39:99-108.

17 Chulichkov AI, Nikolaev AV, Lobanov AI, Guria GT: Threshold activation of blood coagulation and thrombus growth under flow conditions. Matematicheskoe Modelirovanie 2000; 12:75-96.
18 Guzevatykh AP, Lobanov AI, Guria GT: Threshold intravascular blood coagulation as a result of stenosis development (in Russian) Matematicheskoe Modelirovanie 2000; 12:39_ 60.

19 Beltrami E, Jesty J: The role of membrane patch size and flow in regulating a proteolytic feed back threshold on a membrane: possible application in blood coagulation. Math Biosci 2001; 172:1-13.

-20 Leconte M, Martin J, Rakotomalala N, Salin D: Pattern of reaction diffusion fronts in laminar flow. Phys Rev Lett 2003;90:128302.

21 Edwards BF: Poiseuille advection of chemical reaction fronts. Phys Rev Lett 2002;89: 104501 .

22 Abel M, Celani A, Vergni D, Vulpiani A: Front propagation in laminar flows. Phys Rev E 2001;64:046307.

23 Maruyama I, Majerus PW: The turnover of thrombin-thrombomodulin complex in cultured human umbilical vein endothelial cells and A549 lung cancer cells. Endocytosis and degradation of thrombin. J Biol Chem 1985; 260:15432-15438

24 Kogan AE, Strukova SM: Protein C: mechanisms of activation and anticoagulant effect (in Russian). Biokhimiia 1993;58:827-844.

25 Esmon NL, DeBault LE, Esmon CT: Proteolytic formation and properties of gamma-carboxyglutamic acid-domainless protein C. J Biol Chem 1983;258:5548-5553. 